\title{
Commentary on Transabdominal Preperitoneal (TAPP) vs. Totally Extraperitoneal (TEP) for Laparoscopic Hernia Repair: A Meta-Analysis
}

\section{Feng Xian Wei and You Cheng Zhang*}

Department of General Surgery, Lanzhou University Second Hospital, Lanzhou 730030, Gansu Province, People's Republic of China

${ }^{*}$ Corresponding author: You Cheng Zhang, Department of General Surgery, Lanzhou University Second Hospital, Lanzhou 730030, Gansu Province, People's Republic of China, Tel: +86-931-5190524; E-mail: zhangyouchengphd@163.com

Received: June 28, 2017; Accepted: July 25, 2017; Published: August 05, 2017

Citation: Wei FX, Zhang YC (2017) Commentary on Transabdominal Preperitoneal (TAPP) vs. Totally Extraperitoneal (TEP) for Laparoscopic Hernia Repair: A Meta-Analysis. Ann Clin Lab Res Vol.5:No.3:186

\section{Description}

For current less-invasive hernia repair study, operation method and repair material were the most important issues [1]. Our study reviewed the current evidence and concluded an advantage of TAPP in clinical feasibility with comparable efficacy compared with TEP. In their correspondence they raised 2 questions in trial quality assessment and combined model, to which we would reply as follows.

First, we assess the methodological quality based on the methods recommended by Cochrane Handbook, and we also summarized an overall level. As low risks existed in other items, the overall level was mainly judged by randomization, allocation concealment and blinding, which reflected potential bias in the process of selection, performance and detection [2]. Thus, a study with unclear risk of bias in all the 3 major process would be naturally judged as poor-quality with level $\mathrm{C}$ when compared with the others. Besides, the sample size was also one of the considerations. And sensitivity analysis omitting poor-quality was necessary to test the stability of the results especially in random-effects (RE) model, in which the weight of small sample size and poor-quality study was increased than in fix-effects model [3]. After that, they also mentioned that PRISMA statement advice authors to describe how the information on risk of bias was used in data synthesis. Actually, we ensured that both subgroup analysis and sensitivity analysis in our study were related to this issue. Both of them were tried to clarify the influence of potential bias in the mentioned 3 major process as well as other possible bias such as hernia location, surgeon's experience and state.

Second, we thank them to introduce a constructive and very new method of combined model in meta-analysis. It was called quality effects (QE) model or quality adjusted model, and it was developed to improve the conventional RE model, which underestimated the statistical error due to significantly increased heterogeneity [4]. Study applying QE model is subsequently published [5]. As known, the difference model had absolutely different weight distribution for each individual study because of different assumption, and thus led to different combined results. Compared with RE model, QE model complementally took into account the quality assess the results and converted them to quantitative data to adjust the weight distribution [6]. In this reply, we adopted the new method and reported the results in Table $\mathbf{1}$ for comparison purpose. The results did not significantly alter; therefore it demonstrated our study results were reliable and stable.

Table 1 Comparison of meta-analysis results in QE model and RE model.

\begin{tabular}{|l|l|l|l|}
\hline Variables & Pain score & Operation time & $\begin{array}{c}\text { Return to usual activities } \\
\text { time }\end{array}$ \\
\hline Heterogeneity (I2) & $94.55 \%$ & $57.60 \%$ & $34.20 \%$ \\
\hline QE model & SMD & SMD complications & SMD \\
effect size (95\% Cl) & $0.45(-0.57,1.47)$ & $0.15(-0.10,0.39)$ & $-0.08(-0.32,0.16)$ \\
\hline RE model & SMD & SMD & SMD \\
effect size (95\% Cl) & $0.63(-0.20,1.46)$ & $0.12(-0.11,0.35)$ & $-0.12(-0.37,0.13)$ \\
\hline SMD: Standard Mean Difference; RR: risk ratio; QE: Quality Effect; RE: Random Effect & $0.84(0.67,1.06)$ \\
\hline
\end{tabular}

QE model was an improved method based on RE, thus only results of four outcomes in RE model were compared. MetaXL Software (version 4.01, Queensland, Australia) was used, which was available at: www.epigear.com.

I hope the comments and our responses help our readers to understand quality assessment and combined model in meta- analysis, and to further enhance current evidence of operation method choice for laparoscopic hernia repair in practice. 


\section{Acknowledgements}

We would like to thank Dr. Qu and Dr. Ma for their interests in our article, and thank editor for an opportunity to improve our work.

\section{References}

1. Bittner R, Montgomery MA, Arregui E, Bansal V, Bingener J, et al. (2015) Update of guidelines on laparoscopic (TAPP) and endoscopic (TEP) treatment of inguinal hernia (International Endohernia Society). Surg Endosc 29: 1-33.

2. Higgins JP, Altman DG, Gøtzsche PC, Jüni $P$, Moher $D$, et al. (2011) The Cochrane Collaboration's tool for assessing risk of bias in randomized trials. BMJ 343: d5928.
3. Barendregt JJ, Doi SA, MeteXL user guide (version 4.01). Available: http://www.epigear.com/index_files/metaxl.html (Assessed April 7, 2016).

4. Noma H (2011) Confidence intervals for a random-effects metaanalysis based on Bartlett-type corrections. Stat Med 30: 3304-3312.

5. Perera M, Roberts MJ, Doi SA, Bolton D (2015) Prostatic urethral lift improves urinary symptoms and flow while preserving sexual function for men with benign prostatic hyperplasia: A systematic review and meta-analysis. Eur Urol 67: 704-713.

6. Doi SAR, Barendregt JJ, Khan S, Thalib L, Williams GM (2015) Advances in the meta-analysis of heterogeneous clinical trials II: The quality effects model. Contemp Clin Trials 45: 123-129. 